\title{
RADIOLOGY OF STROKE
}

J M Wardlaw

S

troke is a clinical syndrome. ${ }^{1}$ In the investigation of stroke and transient ischaemic attack (TIA) imaging is used to differentiate:

- vascular from non-vascular lesions, such as tumours or infections

- ischaemic from haemorrhagic stroke

- arterial from venous infarction

- and to distinguish anterior and posterior circulation strokes to determine whether a tight carotid stenosis is symptomatic or not.

In the future imaging may be used to show the extent of salvageable tissue in acute stroke before treatment. Imaging should be used to direct management. Investigation should be organised to resolve specific, preferably articulated, management dilemmas. This may include imaging to clarify and guide prognosis.

\section{COMPUTED TOMOGRAPHY}

Computed tomography (CT) will differentiate infarct from haemorrhage up to at least five days after stroke. Recent haemorrhages are high density (white) and usually rounded and space occupying. Infarcts are usually low density (dark) and occupying a vascular territory with some swelling (fig 1). In a patient with a stroke a normal scan excludes a haemorrhage and, in the absence of an alternative, infarction is assumed. ${ }^{2}$

Intravenous contrast is not normally required and may cause confusion.

There is some uncertainty as to how quickly small haemorrhages lose their characteristic whiteness and become isodense and then hypodense compared with normal brain, and so indistinguishable from an infarct. Certainly by 10 days, small haemorrhages will be indistinguishable from infarcts, and we have seen small haemorrhages disappear by seven days. Large haemorrhages remain visible as such for 2-3 weeks.

There is no "optimal" time to image stroke patients with CT and expect to show a definite infarct. Many infarcts do not become visibly hypodense until hours or even a day after the stroke, if ever. ${ }^{3}$ Small infarcts are less likely to be visible than large ones-about $90 \%$ of patients with symptoms of a large cortical infarct (total anterior circulation infarct-TACI) have a visible infarct by 48 hours after stroke compared with about $40 \%$ of patients with lacunar (LACI) or small cortical infarcts (partial anterior circulation infarct-PACI). ${ }^{3}$ Many larger infarcts are visible within six hours though the appearance is subtle and depends on how closely the scan is examined. Observer reliability for specific signs of early infarction (even among experts) is poor (fig 1). ${ }^{45}$ Furthermore, between 10 days and three weeks after stroke, infarcts loose their hypodensity and become isodense with normal brain for several days to two weeks. ${ }^{2}$ As the swelling has also gone by this stage, they may be completely invisible or their true extent impossible to determine. This phase is termed as "fogging". By 2-3 months the infarct has usually become shrunken and is of cerebrospinal fluid density, and so is more readily visible.

The "best time" to image stroke routinely with CT is as soon as possible; nothing is gained by waiting, and much may be lost. In practice the timing of scanning is influenced by what treatments are being considered and available resources. In patients considered candidates for recombinant tissue plasminogen activator (rt-PA), CT scanning is mandatory to exclude intracranial haemorrhage or sizeable infarction before the thrombolytic drug is given. ${ }^{6}$ Currently, in view of the potential hazards, there are good arguments for only considering the use of thrombolysis in a highly organised clinical environment, with appropriate dedicated care pathways established and expert CT scan review available immediately.

In view of the relative lack of harm arising from a few doses of aspirin in patients who turned stroke trial), ${ }^{78}$ clinicians should consider starting aspirin pending the scan if they consider that haemorrhage is unlikely on clinical grounds, and obtain a CT scan the next day. Aspirin may then be stopped if the CT scan shows a haemorrhage. Clinicians and radiologists need to have established guidelines on scanning which reflect the available local resources (see below). 
Figure 1 CT brain scan showing a right hemisphere total anterior circulation infarct $(A)$ at four hours, and (B) at five days after symptom onset. Note on (A) the subtle signs of early infarction: loss of the basal ganglia on the right (white arrow-compare with the left where the caudate and lentiform nuclei are clearly visible), loss of the grey/white matter cortical differentiation (black arrowheads), a little swelling with sulcal effacement (black arrow and compare left side). On day 5 there is obvious hypodensity and massive infarct swelling with midline shift and obstruction of the left lateral ventricle.
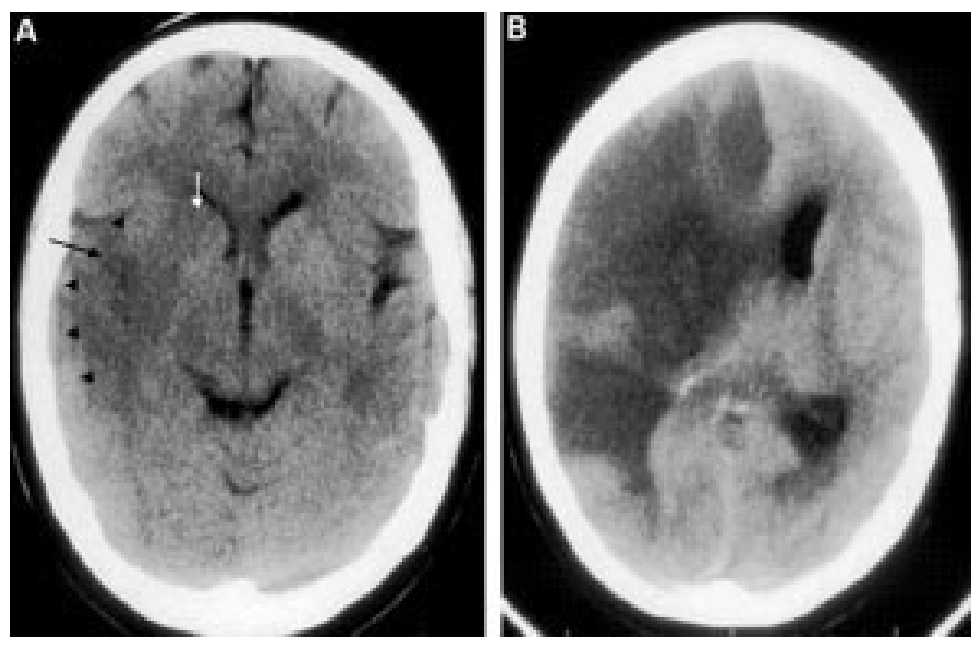

Routine magnetic resonance imaging

\section{Uses of CT in stroke}

- To differentiate vascular from non-vascular disorders

- To differentiate infarct from haemorrhage

- If in doubt, repeat scan a few weeks later without contrast

- Contrast can be misleading and should only be used in special circumstances

\section{Caveats on CT in stroke}

- Identifies all parenchymal haemorrhage with near $100 \%$ accuracy only within 5-7 days of stroke-thereafter small haemorrhages are indistinguishable from infarcts

- Only about $50 \%$ of infarcts ever become visible

- There is no "optimal" time for "seeing" an infarct

- "Seeing" the infarct is not necessary to diagnose ischaemic stroke
The magnetic resonance (MR) appearance of acute haemorrhage is complex, but with the appropriate sequence, acute haemorrhage may be identified correctly even within the first six hours of stroke. ${ }^{2}$ Routine (spin echo) MR sequences remain specific for haemorrhage indefinitely in $90 \%$ of patients. $^{9}$ In the remaining $10 \%$ who have had a definite intracerebral haemorrhage, the diagnostic features (that is, low signal caused by haemosiderin) are not visible on spin echo T2 MR, although cerebromalacea may be visible. Other MR sequences vary in their sensitivity to the presence of haemosiderin. In particular, the now frequently used fast spin echo $\mathrm{T} 2$ and proton density sequences are relatively insensitive whereas gradient echo sequences are the most sensitive (fig 2). If the identification of former haemorrhage is important, it is imperative that the radiologist is told otherwise an inappropriate sequence may be used. With the right sequence, $\mathrm{MR}$ will make this diagnosis in the majority of cases. The same principles of infarct evolution apply to routine MR imaging ( $\mathrm{T} 2$, proton density and $\mathrm{T} 1$ ) as described for CT, including the problems of "fogging" and that a proportion of infarcts will never be visible on routine
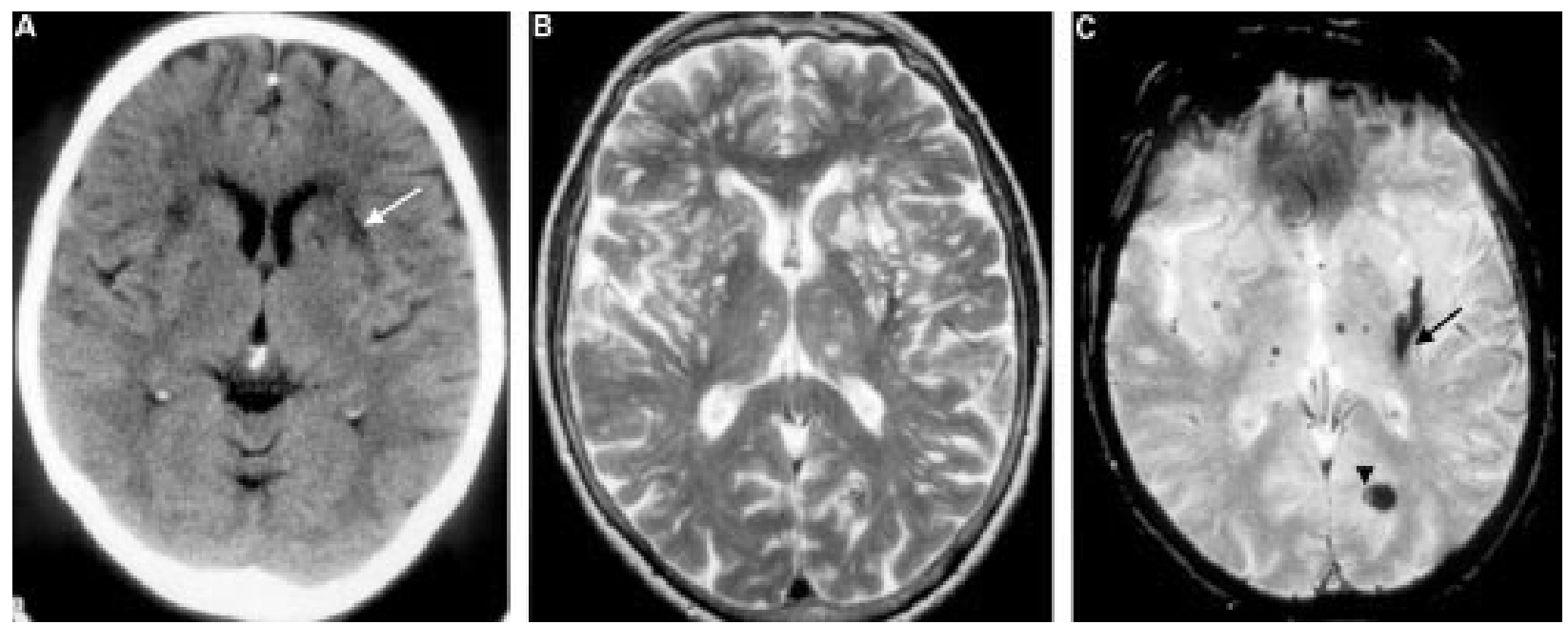

Figure 2 Brain imaging from a 75 year old woman presenting six weeks after a left hemisphere stroke. (A) CT scan. (B) Fast spin echo T2 MR scan. (C) Gradient echo MR scan. Note on the CT scan (A) the lucent areas consistent with small vessel disease. The lucent area in the left hemisphere would be consistent with the symptoms and looks like an infarct. The MR (B, C) obtained on the same day shows not only more small vessel ischaemic changes (white spots) but also haemorrhage (dark area arrowed) in the left lentiform nucleus. The haemorrhage is more readily identified on the gradient echo MR (C) than on the fast spin echo T2 (B). On the latter, old haemorrhage may easily be overlooked. There are also several old microhaemorrhages visible on the gradient echo MR (black dots) and an incidental small calcified lesion in the occipital lobe (arrowhead). 
Figure 3 Cerebral venous thrombosis and infarction (A) pre- and (B) post-intravenous contrast. The scans were obtained at six hours after symptom onset. Note that the hypodensity in the left posterior temporal region is much more developed than for an arterial infarct of the same age (fig $1 \mathrm{~A}$ ), with more clearly defined edges and central haemorrhage (white arrow). After contrast there is central enhancement (white arrow) and a thrombosed transverse sinus is visible (black arrow). The affected territory does not correspond with either the middle cerebral or posterior cerebral arteries, providing a further clue to the venous origin.
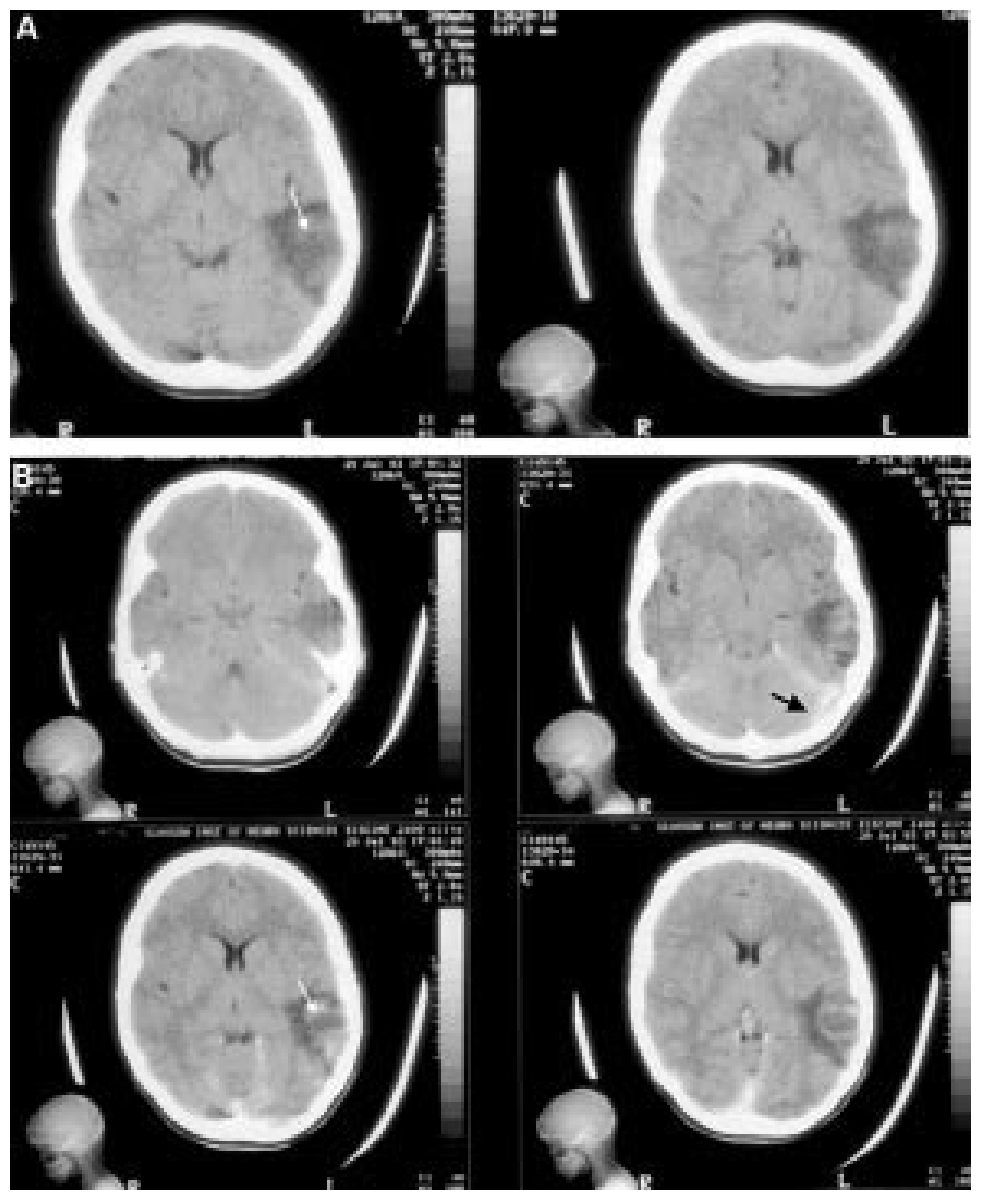

MR. Examples of the evolution of the appearance of infarcts and haemorrhages on MR are also given elsewhere. ${ }^{2}$

\section{"Advanced" magnetic resonance techniques}

Infarcts may be demonstrated on MR with greater sensitivity than CT using certain sequences such as fluid attenuated inversion recovery (FLAIR) or diffusion weighted imaging (DWI). Unfortunately FLAIR also shows many more additional incidental white matter lesions that may simply add to confusion rather than help to identify the new lesion. ${ }^{2}$ Of all techniques diffusion imaging has the greatest sensitivity, but is not specific for infarction. ${ }^{10}$ Encephalitis, multiple sclerosis plaques, and tumours may all appear of increased signal and be mistaken for an infarct by the unwary. On DWI, larger infarcts are visible more often than small ones, and a proportion of patients with a definite stroke never have a visible infarct; some patients who turn out to have a TIA have a relevant visible lesion on DWI when imaged within 24 hours of symptom onset. ${ }^{11}$ Although there is considerable interest in the use of DWI and perfusion $M R$ imaging to identify patients with salvageable tissue, this is still experimental. MR takes longer than CT (so may expose the patient to greater risk of aspiration while supine in the scanner), and may delay the start of treatment as most centres do not have the immediate MR availability required. DWI may be most useful clinically to identify positively the lesion site in patients with minor cortical or lacunar strokes, or to determine whether a patient with a previous infarct and worsening signs has developed a new infarct or not; DWI is likely to be positive up to a week at least after the stroke. ${ }^{12}$ Perfusion imaging with $\mathrm{MR}$ is not in routine use. The most readily available method provides only relative, not absolute, perfusion measurements. Further work is required to improve the interpretation of this technique. MR spectroscopy provides information on metabolites, notably lactate, choline creatine, and $N$ acetyl aspartate (NAA - found in normal functioning neurones). Improved spectroscopic imaging and faster image acquisition times may bring it into wider use, but currently its main application is in stroke research. The exceptions where it may occasionally be useful clinically are to distinguish tumours from infarcts where other imaging is ambiguous (a high choline content is found in tumours, whereas infarcts typically have reduced NAA and normal or reduced choline); or in rare metabolic conditions associated with stroke like MELAS (mitochondrial encephalomyopathy with lactic acidosis and stroke-see below).

\section{Uses of MR in stroke}

- To distinguish haemorrhage from infarct in patients presenting late after stroke-but only if the appropriate sequences are used

- More often shows an ischaemic lesion than CT so may be more useful in "difficult" young patients with suspected stroke

- In "difficult" strokes such as suspected venous infarction or carotid or vertebral dissections, as it may show the vascular anatomy also

\section{Special clinical circumstances}

Venous infarcts are probably underdiagnosed as a cause of stroke (fig 3). Increasing awareness leads to better recognition. ${ }^{213}$ Venous infarcts become hypodense and 
swollen much more rapidly than arterial infarcts and more often contain central areas of haemorrhage. Additional features such as thrombosed venous sinuses (hyperdense sinus pre-contrast, or filling defect in the sinus post-contrast), or opacified paranasal sinuses or mastoids indicating probable infection as the cause of thrombosis, should be sought. MR shows these features more clearly, though they may be visible on CT. Distinguishing tumours from infarcts on CT (or MR) is not usually a problem, but occasionally slow growing tumours such as gliomas can mimic a small cortical infarct by appearing wedge shaped, involving cortex and adjacent white matter, being slightly hypodense, and not enhancing with contrast. Occasionally tumours may also present as a haemorrhage and the bleeding may be extensive enough to obliterate temporarily the underlying neoplasm on the scan. Time is a useful diagnostic tool; repeating the imaging will clarify diagnosis, as infarcts and haemorrhages generally get smaller whereas tumours stay the same or get bigger. Furthermore, patients who initially present with what seems like a straightforward stroke, but who do not behave subsequently as a typical stroke, should have a repeat scan to identify the occasional tumour or other non-vascular lesion. Encephalitis can occasionally mimic stroke, particularly in patients found unwell with diminished consciousness, focal neurology, and no available history of the onset. Imaging, either CT, MR or advanced MR techniques, does not always reliably distinguish between these clinical entities. Diagnosis depends on other assessment. Dissection of the carotid or vertebral artery should be suspected in patients with neck pain and a stroke. MR is best as it may show the vascular and the parenchymal lesion. A typical feature is narrowing of the flow void in the symptomatic carotid or vertebral artery due to a ring or cuff of high signal caused by fresh haemorrhage in the artery wall. Unfortunately this appearance can also be mimicked (more commonly in the carotid than the vertebral artery) by slow flow in the artery above a tight (atheromatous) stenosis, or proximal to a major intracranial arterial occlusion, so some caution is required not to overdiagnose dissection. In view of possible therapeutic implications, intra-arterial angiography should be considered if there is any doubt as to the diagnosis of dissection. CADASIL (cerebral autosomal dominant arteriopathy with subcortical infarcts and leucoencephalopathy) causes prominent subcortical white matter abnormalities which may mimic multiple lacunar infarcts and atrophy, often in a relatively young patient. Imaging is supportive of the diagnosis. MR shows more detail than CT. MELAS (mitochondrial encephalopathy, lactic acidosis, and stroke) presents with stroke in younger patients. On CT or MR cortical infarct-like lesions are visible usually in the posterior temporal or occipito-temporal regions, often bilaterally and not strictly occupying a typical vascular territory.

\section{Who should read the scan?}

MR is complicated and should be left to neuroradiologists or occasionally general radiologists who have a particular interest in neuroimaging. Neurologists and stroke physicians are more used to reading CT scans as the technology has been available for longer, and they should be able to differentiate an infarct from a haemorrhage and to know when a lesion looks non-vascular. Gaining this experience takes time and requires looking closely at as many films as possible. Clinicians should be aware, however, that the signs of early infarction and small haemorrhages can be subtle and overlooked, and calcification may be mistaken for haemorrhage. Therefore clinicians may not be confident to base management decisions on their interpretation of the scan where the identification of early infarction or of subtle haemorrhage is particularly important (for example, before thrombolysis). This is particularly true in the case of trainees in neurology and stroke medicine who simply may not have had the opportunity to see enough scans to gain the requisite experience.

\section{What about the neck arteries?}

Carotid Doppler ultrasound is the simplest, safest, quickest, and best way of assessing the carotid and vertebral arteries to identify atheromatous stenosis or dissection. However, although it looks deceptively easy in experienced hands and when the patient is normal, it is very operator dependent and there are numerous pitfalls for the unwary. Thus it should only be done by properly trained operators with a reasonable throughput of patients to maintain their expertise and where efforts are made to audit the results. Patients with lacunar strokes are less likely than those with cortical infarcts to have a stenosis; about $8 \%$ of lacunar stroke patients will have a tight stenosis in the symptomatic artery. ${ }^{14}$ In some centres ultrasound is the only imaging of the neck done before endarterectomy, while others still rely on intra-arterial angiography for the definitive measurement of stenosis, or use MR or CT angiography. Intra-arterial angiography is risky in patients with symptomatic ischaemic cerebrovascular disease $(4 \%$ permanent stroke, $1 \%$ death) and delays endarterectomy, and $\mathrm{MR}$ and $\mathrm{CT}$ angiography are no better than Doppler ultrasound in competent hands (though they may be alternatives where ultrasound expertise is lacking). Whatever is used, it is important that the imaging is reported by radiologists with an interest in carotid disease.

\section{Use of guidelines and organising local investigations}

Stroke is such a common condition that an average general hospital will admit one or two cases per day. Thus the investigation of stroke must be well organised, otherwise a backlog develops resulting in delayed, inefficient, and suboptimal patient management. Imaging resources (either money or scanner time) are not infinite. Inappropriate resource use also displaces patients with other (possibly more needy) diagnoses, and creates conflict and friction between different clinical departments and radiological service providers. These difficulties may be particularly true in hospitals where there is a large mixed general body scanning workload. Imaging resources in most parts of the country are still well below those required by the local populations. Various bodies have issued guidelines for the investigation of stroke. ${ }^{15}$ Individual hospitals will need to determine how best to apply these guidelines in light of their own resources. Good communication between clinical and radiological departments is absolutely fundamental to this process.

\section{References}

1 Hatano S. Experience from a multicentre stroke register: a preliminary report. Bull WHO 1976;54:541-53.

2 Wardlaw JM. What pathological type of stroke is it? In: Warlow CP, et al, eds. Stroke, a practical guide to management, 2nd ed. Oxford: Blackwell Science (in press).

In depth and comprehensive coverage of brain imaging in stroke set out in a practical and logical order with lots of examples. There are important additions to the second edition so read that in preference to the first, as there has been an explosion of information on neuroimaging since the first edition appeared. 
3 Wardlaw JM, Lewis SC, Dennis MS, et al. Is visible infarction on computed tomography associated with an adverse prognosis in acute ischaemic stroke? Stroke 1998;29:1315-19.

4 Wardlaw JM, Dorman PJ, Lewis SC, et al. Can stroke physicians and neuroradiologists identify signs of early cerebral infarction on CT? J Neurol Neurosurg Psychiatry 1999;67:651-3.

5 von Kummer R, Bozzao L, Manelfe C. Early CT diagnosis of hemispheric brain infarction. Berlin: Springer, 1995.

- A very useful guide to early infarct signs on CT with lots of excellent examples and follow up scans so that the reader can see how the infarct developed.

6 Wardlaw JM, del Zoppo G, Yamaguchi T. Thrombolytic therapy in acute ischaemic stroke. Part 1. Thrombolysis versus control. In: Warlow C, Van Gijn J, Sandercock P, eds. Stroke module of the Cochrane Database of Systematic Reviews, [updated 10 October 1999]. Available in The Cochrane Library [database on disk and CD-ROM]. The Cochrane Collaboration; Issue 4. Oxford: Update Software; 1999. Updated quarterly-third substantive revision.

- Full details of all the available evidence on thrombolysis in acute ischaemic stroke. A must for anyone considering either participating in a new trial or implementing a routine thrombolysis service.

7 The International Stroke Trial Collaborative Group. The international stroke trial (IST): a randomised trial of aspirin, subcutaneous heparin, both or neither among 19435 patients with acute ischaemic stroke. Lancet 1997;349:1569-81.

8 Chen ZM, Sandercock PAG, Pan HC, et al, on behalf of the CAST and IST collaborative groups. Indications for early aspirin use in acute ischaemic stroke. A combined analysis of 40,000 randomised patients from the Chinese acute stroke trial and the international stroke trial. Stroke 2000;31:1240-9.

- A must for all doctors treating acute stroke-the full details on the risks and benefits of aspirin.

9 Wardlaw JM, Statham PFX. How often is haemosiderin not visible on routine MRI following traumatic intracerebral haemorrhage. Neuroradiology 2000;42:81-4.

10 Powers WJ. Testing a test. A report card for DWI in acute stroke. Neurology 2000;54:1549-51.

- A very well balanced critique of the enthusiastic literature on diffusion MR imaging, pointing out some problems.

11 Keir SL, Wardlaw JM. A systematic review of diffusion and perfusion MR imaging. Stroke 2000;31:2723-31.

- The detailed data to back up the comments in reference 10! Both 10 and 11 are essential for anyone interested in either routine use of advanced MR in stroke, or its use in research.

12 Wardlaw JM, Armitage P, Dennis MS, et al. The use of diffusion-weighted magnetic resonance imaging to identify infarctions in patients with minor strokes. J Stroke Cerebrovasc Dis 2000;9:70-5.

13 Bakaç G, Wardlaw JM. Problems in the diagnosis of intracranial venous infarction. Neuroradiology 1997,39:566-70.

14 Mead GM, Wardlaw JM, Lewis SC, et al. Can simple clinical features be used to identify patients with severe carotid stenosis on Doppler ultrasound? J Neurol Neurosurg Psychiatry 1999;66:16-19.

15 Scottish Intercollegiate Guidelines Network (SIGN). Management of patients with stroke. 1. Assessment, investigation, immediate management and secondary prevention. Edinburgh: SIGN, 1997.

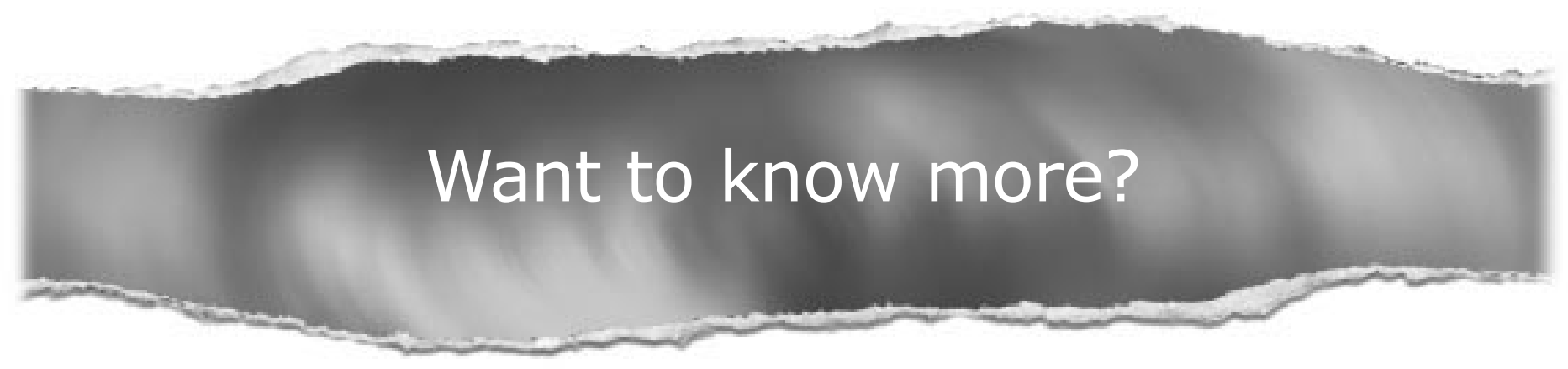

Data supplements

Limited space in printed journals means that interesting data and other material are often edited out of articles; however, limitless cyberspace means that we can include this information online.

Look out for additional tables, references, illustrations.

www.jnnp.com 